\title{
Contrasting heterozygosity-fitness correlations between populations of a self-compatible shrub in a fragmented landscape
}

\author{
Juan P. González-Varo • Abelardo Aparicio • \\ Sébastien Lavergne $\cdot$ Juan Arroyo • \\ Rafael G. Albaladejo
}

\begin{abstract}
The mechanisms underlying heterozygosity-fitness correlations (HFCs) are subject of intense debates, especially about how important population features such as size or degree of isolation influence HFCs. Here, we report variation in HFCs between Large and Small populations of a self-compatible shrub (Myrtus communis) occurring within an extremely fragmented landscape. In each of the five study populations, we obtained data on both heterozygosity and fitness for 9-12 maternal families (i.e. offspring from the same mother plant). Whereas heterozygosity explained most of the variance (60-86 \%) in growth rate of seedling families within Large populations, this relationship was absent within Small populations. Our results suggest that inbreeding may explain the observed HFCs within Large populations, and that different genetic processes (such as genetic drift and/or selection) could have overridden HFCs within Small populations. While it is difficult to draw general conclusions from five populations, we think our results open new research perspectives on how different genetic processes underlie variation in HFCs under different population contexts. Our
\end{abstract}

Electronic supplementary material The online version of this article (doi:10.1007/s10709-012-9655-8) contains supplementary material, which is available to authorized users.

J. P. González-Varo ( $₫)$

Estación Biológica de Doñana (EBD-CSIC), Avda. Américo

Vespucio s/n, Isla de La Cartuja, 41092 Sevilla, Spain

e-mail: juanpe@ebd.csic.es

A. Aparicio - J. Arroyo · R. G. Albaladejo

Departamento de Biología Vegetal y Ecología, Universidad de Sevilla, Sevilla, Spain

S. Lavergne

Laboratoire d'Ecologie Alpine, UMR 5553 CNRS, Université

Joseph Fourier, Grenoble, France study also points to a need for further attention on the complex relationships between heterozygosity in self-compatible plants and their progeny in relation to mating system variation. Finally, our results provide interesting new insights into how population genetic diversity is maintained or lost in a highly fragmented landscape.

Keywords Habitat fragmentation · Inbreeding depression · Mixed mating systems $\cdot$ Myrtus communis $\cdot$ Population genetics

\section{Introduction}

The existence of positive correlations between heterozygosity and fitness has been documented several times (reviewed in Mitton and Grant 1984; Britten 1996; David 1998). However, questions regarding the importance and especially the generality of heterozygosity-fitness correlations (HFCs) have generated profound debates to date (see Britten 1996; David 1998; Hansson and Westerberg 2002; Szulkin et al. 2010). On the one hand, meta-analyses have shown that HFCs are not as common as previously thought (Britten 1996; Coltman and Slate 2003; Chapman et al. 2009), and also that HFCs can be highly variable among taxa and even among samples (populations and cohorts) within the same species (e.g. Ledig et al. 1983; David et al. 1997). On the other hand, our capacity for detecting HFCs may depend on the type of codominant molecular marker that is used to estimate heterozygosity (Pogson and Zouros 1994; Thelen and Allendorf 2001; see also Karl and Avise 1992).

Two main types of mechanisms are thought to generate HFCs: (1) functional overdominance of the marker loci per se, known as direct effect (possible with allozymes since 
they are linked to metabolism and physiology); and (2) associative overdominance between marker loci and fitness loci, which may occur either if the marker loci are closely linked with fitness loci (local effect) or if the marker loci represent heterozygosity on a genome-wide basis (general effect; i.e. inbreeding) (see David 1998; Hansson and Westerberg 2002; Szulkin et al. 2010). The direct effect hypothesis was considered as a main mechanism underlying HCFs until the 1990s, when the use of allozyme markers was widespread (see reviews Mitton and Grant 1984; Mitton 1997; David 1998). Thereafter, HFCs have also been observed with non-coding neutral markers (e.g. microsatellites) and, consequently, the two types of associative overdominance have captured the debate as alternative hypotheses (Zouros 1993; Bierne et al. 2000; Hansson and Westerberg 2008; Amos and AcevedoWhitehouse 2009; Szulkin et al. 2010).

The magnitude of associative overdominance, and thus of HFCs, is mostly determined by the level of inbreeding within populations (Szulkin et al. 2010), although other processes such as genetic drift, immigration and selection can strengthen associative overdominance through linkage disequilibrium between loci (Hansson and Westerberg 2002, 2008; Grueber et al. 2008; Szulkin et al. 2010). Nevertheless, a recent review on empirical evidence in animals has shown that the predictive power of marker loci (exclusively microsatellites) to fitness traits is expected to be very low ( $\sim 1 \%$ of variance; Chapman et al. 2009) under realistic levels of inbreeding in populations unable to self-fertilization (see also Szulkin et al. 2010). It has to be noted, however, that most empirical studies on HFCs in the last two decades have been conducted on animals with separate sexes (Coltman and Slate 2003; Chapman et al. 2009; Szulkin et al. 2010), so there is a strong publication bias that may limit a broader understanding of their prevalence (see also Supplementary Material in Grueber et al. 2008). In hermaphroditic self-compatible plants, inbreeding may occur through outcrossing between close relatives (i.e. biparental inbreeding), but more importantly through selfing (i.e. uniparental inbreeding; Goodwillie et al. 2005), which could trigger the emergence of HFCs (see Britten 1996). Indeed, in his review, Britten (1996) found stronger HFCs in plants than in animals, supporting this idea. Another interesting feature of selfing organisms is that heterozygosity in progeny may strongly depend on maternal heterozygosity.

Previous works [from Mitton and Grant (1984) to Grueber et al. (2008) and Chapman et al. (2009)] have suggested that comparisons among populations differing in important features such as size, degree of isolation, age or inbreeding, can provide valuable settings for interpreting the underlying mechanisms of HFCs. In this context, long-term fragmented plant populations can offer ideal opportunities to assess variation in HFCs as they may differ in contemporary mating patterns (outcrossing/selfing rates) and may exhibit increased linkage disequilibrium, reduced genetic diversity and may have purged some genetic load through a long history of inbreeding (Ellstrand and Elam 1993; Byers and Waller 1999; Aguilar et al. 2008; Grueber et al. 2008; Eckert et al. 2010).

Here, we report variation in HFCs among progenies of a self-compatible shrub (Myrtus communis L.) in Large and Small populations occurring within a historically fragmented landscape. In the same maternal families (i.e. offspring from the same mother plant), we used allozymes as genetic markers to estimate their mean heterozygosity and, further, conducted a greenhouse experiment in order to evaluate their fitness in terms of germination, growth and survival. We found in a previous study that the progeny of $M$. communis from the populations studied here were generated by a mixture of self- and cross-fertilization events, with mean outcrossing rates of populations ranging from 13 to $72 \%$ (González-Varo et al. 2010). The fact that outcrossing rates were not correlated with population size, but rather attributable to differences in pollinator faunas, provides us with the opportunity to distinguish between the role of historical (long history of inbreeding, genetic drift and/or selection) and contemporary (the mating patterns) genetic processes on the expression of HFCs.

\section{Materials and methods}

Study species, area and populations

The Mediterranean myrtle (Myrtus communis) is a common sclerophyllous shrub that grows up to $4 \mathrm{~m}$ high and inhabits fertile lowlands across the Mediterranean Basin. Flowers (white open dish-shaped corolla) are hermaphroditic and insect-pollinated (mostly by bees and flies), while fruits are dark-blue berries that contain $\sim 5$ seeds each of $\sim 12 \mathrm{mg}$ (González-Varo et al. 2009). The species is selfcompatible and shows a mixed-mating system (GonzálezVaro et al. 2009, 2010).

The Guadalquivir Valley (western Andalusia, southern Spain) is a large (ca. 21,000 $\mathrm{km}^{2}$ ) lowland area that has been intensively cultivated for centuries. As a consequence of a long-term transformation of natural habitats, only $\sim 1 \%$ of Mediterranean woodland cover remains at present in over 530 forest patches (mainly composed of cork oak Quercus suber, holm oak Q. ilex subsp. ballota and/or stone pine Pinus pinea) scattered throughout the Valley (for further details see Aparicio 2008). In this study we focused on five myrtle populations occurring in five of such patches: two large ( $>2,000$ individuals) and three small ( $\leq 70)$ populations (hereafter, Large and Small populations, 


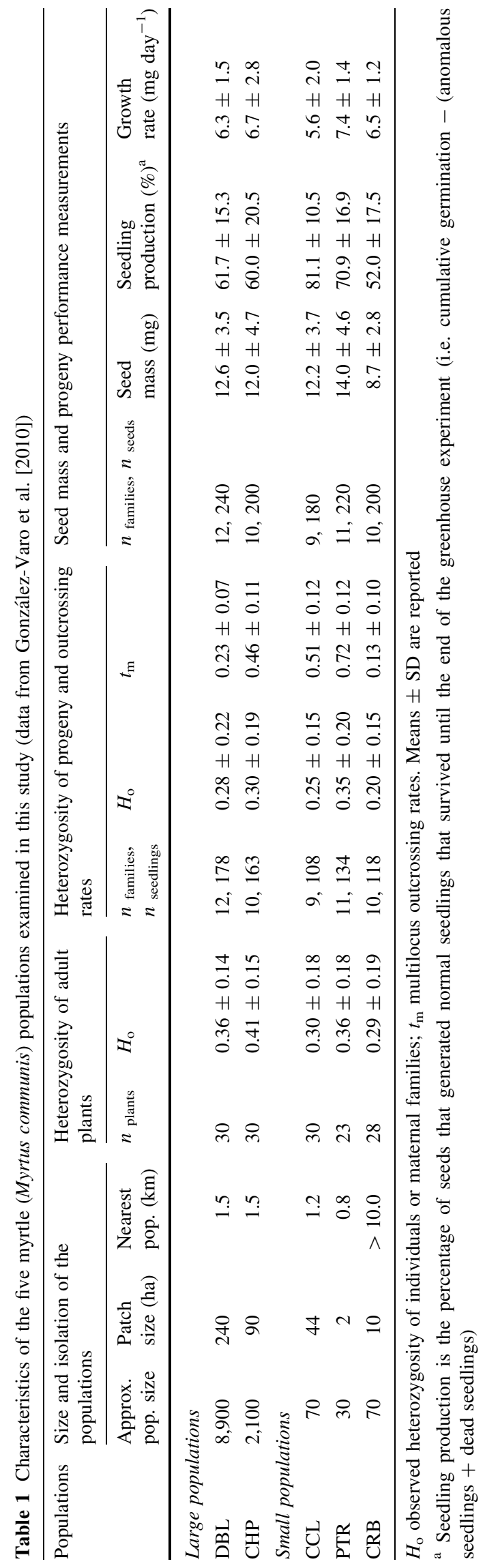

respectively; for a map see Fig. S1 in González-Varo et al. 2010). Table 1 summarizes the characteristics of these five populations, including genetic parameters and the two fitness traits used to test HFCs.

\section{Sampling design}

In a previous study (González-Varo et al. 2010), we obtained data on both allozyme variability and fitness from naturally pollinated progeny arrays belonging to the five studied populations. In the present study, we address the relationships between allozyme heterozygosity and fitness at the family level by averaging values obtained for each group of seeds/seedlings belonging to the same mother plant (i.e. family); working at the family level allows us to test the relationships between heterozygosity and seed germination, which is a fitness trait that cannot be tested at the individual level because of the destructive nature of the protein/DNA extraction protocols. We gathered data on allozyme heterozygosity and fitness for a total of 52 maternal families (9-12 families per population; $n=701$ genotyped seedlings and 1,040 seeds tested in a greenhouse experiment). For progeny sampling, 20 ripe fruits were collected from throughout the canopy of each of 12 mother plants per population during November 2006 (totalling 60 plants and 1,200 fruits). Fruits were dissected and from each fruit two seeds were randomly separated; one for allozyme analyses and the other for the greenhouse experiment. For each mother plant, the mean seed mass was obtained by weighing 20 seeds from the remaining seed pool ( $n=1,200$ seeds).

\section{Allozyme heterozygosity}

The 20 seeds per mother plant sampled for allozyme analysis were germinated in Petri dishes and germination percentages of well over $90 \%$ were obtained whenever seeds were not fungus infected (González-Varo et al. 2010). Preparation of the seedling material, gel electrophoresis and enzyme staining procedures were performed as described by González-Varo et al. (2010). Four loci were resolved with a total of 11 alleles: two in each of the $I d h-2$ and Pgm-1 loci, three in the Ugpp-2 locus, and four in the Pgi-2 locus. These loci were the most polymorphic loci out of 12 loci resolved for the species (see details in González-Varo et al. 2010). Overall, 710 seedlings were sampled $(n=8-19$ per mother plant; mean $\pm \mathrm{SD}=$ $13.7 \pm 2.4$ ) from $9-12$ plants per population (total 52 mother plants) and multilocus allozyme profiles were successfully generated for 701 (sample sizes are shown in Table 1); some families and individual seedlings were lost by fungal infection. Family-level heterozygosity $\left(H_{\mathrm{o}}\right.$ : proportion of heterozygous loci) was estimated by averaging values obtained for each group of seedlings of the same 
mother plant. Mean $H_{\mathrm{o}}$ values of families per locus (all the populations pooled) were 0.371 (Idh), 0.032 (Pgm), 0.400 (Ugpp) and 0.307 (Pgi). We used the software MLTR v. 3.3. (Ritland 2002) to estimate the genotypes of the mother plants from the diploid multilocus genotypes of the seedlings.

Fitness measurements

Progeny performance was evaluated in a common greenhouse environment using the 20 seeds per mother plant sampled for this purpose. On December 2006, a total of 1,200 seeds from the five study populations (12 mother plants per population $\times 20$ seeds per mother) were individually sowed in trays of 60 pots $(5 \times 5 \mathrm{~cm}$ and $17 \mathrm{~cm}$ depth), as described by González-Varo et al. (2010). Seed germination and seedling mortality were monitored regularly until the end of the experiment (February 2008; total $=406$ days since sowing). Then, the seedlings were harvested and dried at $60{ }^{\circ} \mathrm{C}$ for $72 \mathrm{~h}$ and dry biomass was then measured. Some seedlings grew anomalously, i.e. they weighed $\sim$ fourfold less $($ mean $=0.66 \mathrm{~g}$ ) and bore smaller leaves than normal seedlings (mean $=2.42 \mathrm{~g}$; see details in González-Varo et al. 2010). These seedlings were thus classified as 'anomalous', because it is reasonable to expect that they have much lower chances of survival in the wild.

For each family, we calculated two fitness parameters. The first parameter, 'seedling production', was calculated as the percentage of seeds of each family that generated normal seedlings that survived until the end of the experiment (i.e. seedling production $(\%)=$ cumulative germination - [anomalous seedlings + dead seedlings]). Although this parameter combined germination, survival and viability (i.e. normal growth), it was mostly determined by accumulated germination across populations $(r=0.75-0.97, P<0.005$, $n=9-12)$. We did not analyze mortality and anomalous growth separately because both measurements were extremely variable either among or within populations (GonzálezVaro et al. 2010), with most families showing zero values; low mortality rates can be explained by optimal growth conditions in the greenhouse. The second parameter, 'growth rate' (biomass growth rate; $\mathrm{mg} \mathrm{day}^{-1}$ ), was calculated for each seedling (and for each family averaging seedling values) by dividing its dry biomass by its growth time $\left(t_{\text {growth }}=t_{\text {total }}-t_{\text {emergence }}\right.$; being $t_{\text {total }}$ the total number of days of the experiment [ 406 days] and $t_{\text {emergence }}$ the number of days until seedling emergence). Although seedling dry biomass and growth rate were highly correlated $\left(r^{2}=0.99\right.$, $P<0.001, n=52$ ), we finally used the latter to control for the differences among seedlings/families in emergence date. No significant levels of collinearity were observed between the two fitness variables either in any particular population or overall (Pearson's $r<10.271, P>0.45$ ).
Statistical analyses

For the subset of 52 maternal families (9-12 per population) for which data on both allozyme variability and progeny performance were available, correlations between mean observed heterozygosity $\left(H_{\mathrm{o}}\right)$ and the two fitness parameters (seedling production and growth rate) were investigated through Pearson's correlations. Correlations were performed for each population and also for each population type (Large and Small populations). We used partial correlations to ensure that the effects of heterozygosity on progeny performance measurements were independent of the effects of seed mass (i.e. maternal effects; Roach and Wulff 1987; see also Byers and Waller 1999).

We performed Pearson's correlations in order to check the consistency across loci in heterozygosity because a general effect would imply correlation between heterozygosities measured across different loci (i.e. identity disequilibria; see Balloux et al. 2004; Szulkin et al. 2010). We also used Pearson's correlations to check for variation among populations in the relationships between $H_{\mathrm{o}}$ of mother plants and $H_{\mathrm{o}}$ of seedling families (Mitton et al. 1993), whereas we used Spearman's rank correlation $\left(r_{\mathrm{s}}\right)$ to check for the effect of the population outcrossing rate on the strength of such relationships. All analyses were performed with the software STATISTICA v.6 (StatSoft 2001).

\section{Results}

Heterozygosity-fitness correlations

Whereas the mean growth rate of seedling families was highly correlated with $H_{\mathrm{o}}$ in the Large populations ( $r=$ 0.774-0.928, $P<0.05$ ), correlations were non-significant and close to zero $(r<10.201, P>0.3)$ in the Small populations (see Table 2; Fig. 1). Remarkably, $H_{\mathrm{o}}$ explained $60-86 \%$ of variance in the growth rate of seedling families within Large populations. Seedling production of families was not significantly correlated with multilocus heterozygosity $\left(H_{\mathrm{o}}\right)$ in any of the five study populations (Table 2 ).

\section{Consistency across loci}

In order to check for locus-specific effects, we performed additional partial correlations between seedling growth rate and $H_{\mathrm{o}}$ at different number and combinations of loci (from 1 to 4; see details in Fig. S1 in Supporting Information). Results showed that (1) again, significant correlations between $H_{\mathrm{o}}$ and growth rate occurred only in Large populations; (2) such significant correlations were detected either using single locus or multilocus $H_{\mathrm{o}}$ based on groups of 2 and 3 loci; and that (3) in Large populations, the 
Table 2 Pearson's partial correlation coefficients between mean observed heterozygosity $\left(H_{\mathrm{o}}\right)$ and mean values for two fitness measurements in families of Myrtus communis belonging to Large and Small populations (seedling production is defined in Table 1)

\begin{tabular}{lrcc}
\hline Populations & $n$ & Seedling production & Growth rate \\
\hline Large populations & 22 & $0.139 \mathrm{~ns}$ & $\mathbf{0 . 8 1 0 * * *}$ \\
DBL & 12 & $0.169 \mathrm{~ns}$ & $\mathbf{0 . 9 2 8} * * *$ \\
CHP & 10 & $0.139 \mathrm{~ns}$ & $\mathbf{0 . 7 7 4}$ \\
Small populations & 30 & $-0.095 \mathrm{~ns}$ & $0.179 \mathrm{~ns}$ \\
CCL & 9 & $-0.097 \mathrm{~ns}$ & $0.299 \mathrm{~ns}$ \\
PTR & 11 & $-0.468 \mathrm{~ns}$ & $-0.029 \mathrm{~ns}$ \\
CRB & 10 & $-0.173 \mathrm{~ns}$ & $-0.113 \mathrm{~ns}$ \\
\hline
\end{tabular}

Each partial correlation coefficient was calculated after controlling for the effect of seed mass, i.e. maternal effects. For clarity, significant correlation coefficients are in bold

ns $P>0.05 ; * P<0.05 ; * * * P<0.001$

higher the number of loci used to estimate $H_{\mathrm{o}}$, the higher the values of the correlation coefficient (Fig. S1).

We also tested whether a multiple regression incorporating specific effects for each locus explained more variance on growth rate of seedling families than a simple regression incorporating multilocus heterozygosity, which allows detecting significant local effects (as described in Szulkin et al. 2010). Differences between models were non-significant (adjusted $r^{2}=0.64$ and 0.61 for the simple and multiple regression, respectively; $F_{3,17}=1.1, P>0.05$ ), thus not supporting the local effect hypothesis (linkage disequilibrium) as the mechanism behind HFCs within the Large populations (see more results and details in Table S1).

Finally, correlations between $H_{\mathrm{o}}$ of families estimated with different pairs of loci were in general positive within the Large populations (mean $r=0.482-0.581$, mean $P=0.093-0.122)$, with some significant $(P<0.05)$ and marginally significant $(P<0.10)$ correlations (see results in Table S2). This pattern was also found in the Small population PTR (mean $r=0.635$, mean $P=0.078$ ). However, in the two other Small populations all correlations were non-significant (mean $r=0.193-0.331, P=$ 0.402-0.532; see Table S2).

Effects of maternal heterozygosity and outcrossing rates

As expected, heterozygous mothers tend to produce more heterozygous offspring. Observed heterozygosity $\left(H_{\mathrm{o}}\right)$ of mother plants was positively associated with mean $H_{\mathrm{o}}$ at the family level across populations $(r=0.555-0.966$, $P<0.05$ in DBL, CHP and CRB, and $P=0.05-0.10$ both in CCL and PTR; see Table S3). Consequently, the results of HFCs using $H_{\mathrm{o}}$ values of mother plants were very similar to those using $H_{\mathrm{o}}$ at the family-level (Table S3). However, the highest and most significant correlations
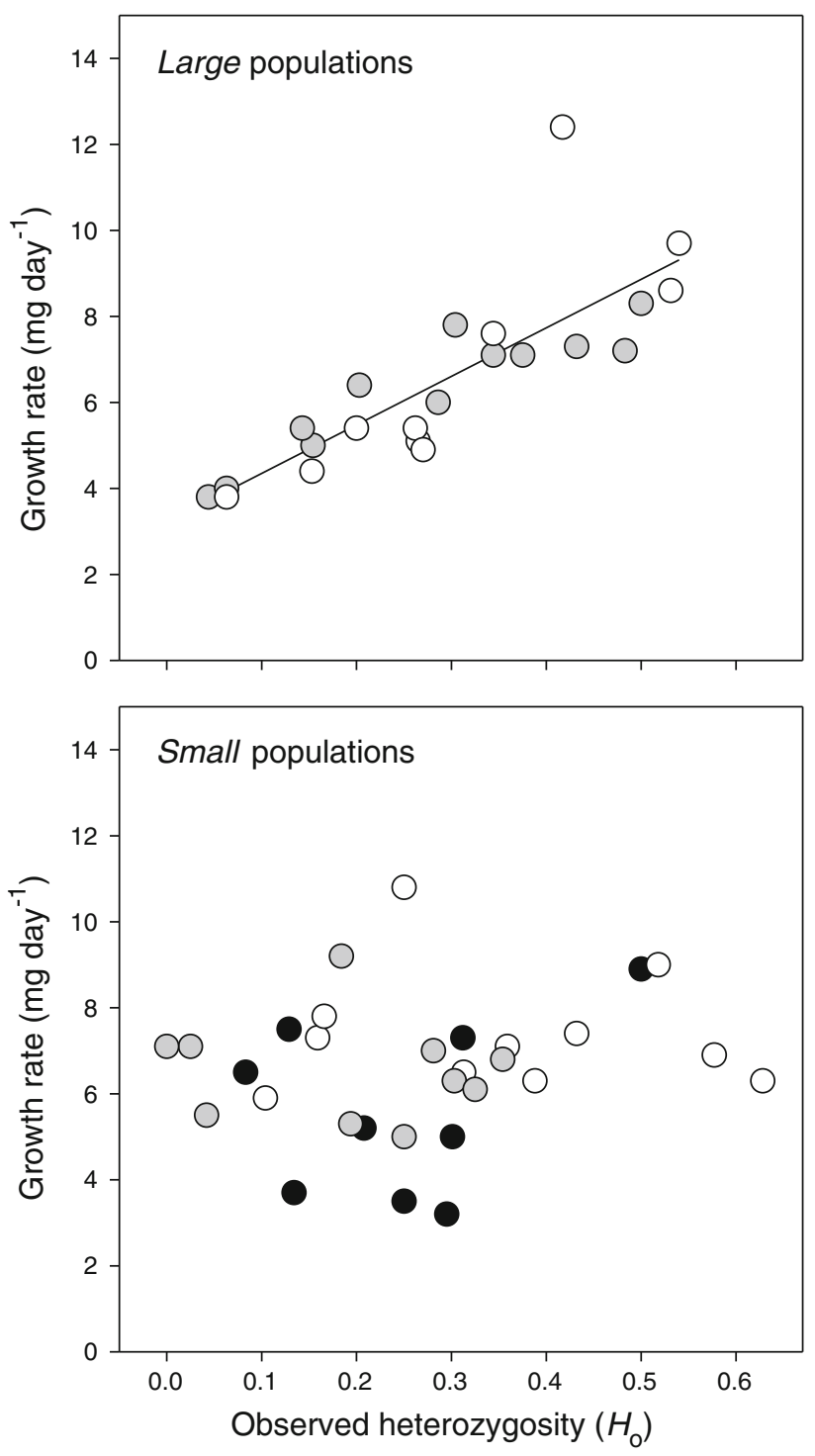

Fig. 1 Relationship between the mean observed heterozygosity $\left(H_{\mathrm{o}}\right)$ and the mean growth rate of Myrtus communis seedling families belonging to two Large (grey circles, DBL; white circles, $\mathrm{CHP}$ ) and three Small populations (black circles, CCL; white circles, PTR; grey circles, CRB). The line in the upper panel represents the least squares regression line for the two large populations pooled

between $H_{\mathrm{o}}$ of mother plants and mean $H_{\mathrm{o}}$ at the family level were found in those populations showing the highest levels of selfing (i.e. the lowest outcrossing rates $t_{\mathrm{m}}$; see Fig. 2). This result indicated that $H_{\mathrm{o}}$ of seedling families was strongly determined by $H_{\mathrm{o}}$ of their mother plants in populations with high selfing rates, regardless of population sizes (see Fig. 2).

\section{Discussion}

Intraspecific variation in heterozygosity-fitness correlations (HFCs) may provide valuable evidences on underlying 


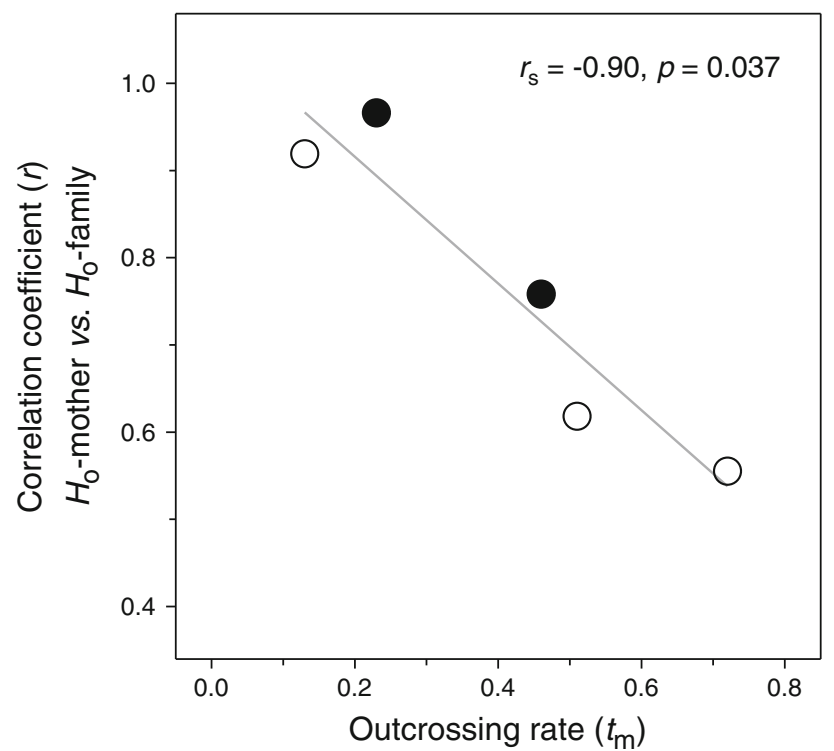

Fig. 2 Relationship between the outcrossing rates $\left(t_{\mathrm{m}}\right)$ and the correlation coefficient between heterozygosity $\left(H_{\mathrm{o}}\right)$ of mother plants and mean heterozygosity of their progenies (families) in the five studied populations. Black circles represent Large populations; white circles Small populations. The line represents the least squares regression line

mechanisms and their implications on population genetics and performance (Mitton and Grant 1984; Grueber et al. 2008; Chapman et al. 2009). Surprisingly, empirical studies investigating HFCs within several populations of the same species are still very scarce (Grueber et al. 2008). Our study shows that HFCs were inconsistent across myrtle populations which concurs with the seminal study by Ledig et al. (1983), who found that, only in certain populations (the oldest stands), the growth rate of Pinus rigida trees was explained by allozyme heterozygosity (up to $\sim 80 \%$ of variance).

More importantly, we found that the occurrence of HFCs was associated with the type of myrtle population in relation to anthropogenic habitat fragmentation (Large and Small). Contrary to the expectations, we did not detect HFCs within small-and perhaps bottlenecked-populations (see Bierne et al. 2000; Hansson and Westerberg 2002; Grueber et al. 2008), but rather within large populations. Two questions arise when looking at these results: what mechanism explains the observed HFCs within Large populations and what genetic process is behind their absence within Small ones? Because the Small populations studied were either predominantly outcrossed (PTR: $t_{\mathrm{m}}=0.72$ ), half-outcrossed (CCL: $\left.t_{\mathrm{m}}=0.51\right)$ or predominantly selfed (CRB: $t_{\mathrm{m}}=0.13$ ), it would seem unlikely that the pattern of HFCs among population types was caused by mating system variation per se (see Table 1; Fig. 2).

A plausible hypothesis to explain the observed HFCs, and more consistent with early HFCs studies, would be that allozyme heterozygosity had positive effects on seedling growth rate (i.e. functional overdominance; direct effect hypothesis). In fact, several studies that examined relationships between allozyme heterozygosity and growth rate-most of them in large populations of marine animals, where inbreeding is expected to be rare-reported strong positive results (see chapter 7 in Mitton 1997). In our case, the direct effect hypothesis would then require that inbreeding depression effects in Small populations have masked the incremental advantages of enzyme heterozygosity. However, this is quite unlikely given the similar mean seedling growth rates found in Large and Small populations (see Table 1).

There is some evidence pointing that inbreeding (i.e. associative overdominance; general effect hypothesis) was behind of HFCs within Large populations, such as the lack of significant locus-specific effects (Table S1) and the trend towards higher HFCs when using a larger number of marker loci to estimate $H_{\mathrm{o}}$ (see Fig. S1). Moreover, inbreeding would imply positive correlations between heterozygosities measured across different subsets of loci (identity disequilibria; see Balloux et al. 2004; Szulkin et al. 2010) and, despite the small number of markers used, we found a general trend of positive associations between $H_{\mathrm{o}}$ estimated with different halves of the loci examined within Large populations (see Table S2). It could be argued that the absence of HFCs in Small populations is consequence of a long history of inbreeding, which have purged the genetic load affecting seedling growth in some maternal lines/populations (see Dudash et al. 1997; Byers and Waller 1999) but, again, the similar seedling growth rates found in Large and Small populations do not support this idea (Table 1). Alternatively, the finding of nearly-significant identity disequilibria in only one (PTR) out of three Small populations (see Table S2) along with the strong among-population variation in outcrossing rates (thus, in $H_{\text {o-maternal }}-H_{\text {o-family }}$ correlations; Fig. 2$)$ suggest that different genetic processes-such as genetic drift and/or selection-may be behind the absence of HFCs within the Small populations of our study (see Grueber et al. 2008; Mueller et al. 2010).

Both the direct effect and the general effect hypotheses may also be supported by the fact that significant associations with $H_{\mathrm{o}}$ were found for seedling growth but not for seedling production. Growth rate has been considered as one of the most important trait to assess and detect HFCs (see Ledig et al. 1983; Mitton and Grant 1984; Britten 1996; David 1998) because it represents an integrated lifehistory trait, likely involving many functional loci (all of them being susceptible to deleterious mutations). Conversely, germination (i.e. to germinate or not), survival and anomalous growth could depend on just one or a few deleterious alleles of large effect (lethal or semilethal) in 
homozygosity (Charlesworth and Charlesworth 1999; Charlesworth and Willis 2009). Regarding the general effect hypothesis, the difference between the fitness parameters tested here (seedling production and growth rate) might arise from the number and type of deleterious alleles responsible for inbreeding depression. This idea is very consistent with a recent study (González-Varo and Traveset 2010) testing among-family variation in inbreeding depression of $M$. communis progenies produced by controlled crossings (self- and cross-pollinations). Beyond seedling growth in itself, it is expected that seedlings exhibiting slow growth rates will have much lower chances of survival under natural conditions (see González-Varo et al. 2010, 2012). Hence, our results can explain to some extent the maintenance or loss of genetic diversity in fragmented myrtle populations (see Albaladejo et al. 2009) since the most heterozygous families were also the most vigorous within the Large but not within the Small populations studied. Besides the fact that variability in heterozygosity and growth rate is low between populations $\left(\mathrm{CV}_{H \mathrm{o}}=17 \%, \mathrm{CV}_{\text {growth }}=9 \%\right)$ but high within them $\left(\mathrm{CV}_{H \mathrm{o}}=43-67 \%, \mathrm{CV}_{\text {growth }}=18-34 \%\right)$, our results can also explain why we did not detected significant HFCs at the population level in a previous study (González-Varo et al. 2010).

\section{Concluding remarks}

Demonstrating HFCs usually requires large sample sizes, and it is thus particularly striking that we were able to find strong correlations with the modest number of loci and families analyzed in this study. On the one hand, self-fertilization in our study species allows high levels of inbreeding, currently considered the main factor behind HFCs (Szulkin et al. 2010). On the other hand, information on each family represents the mean of many seed and seedlings, so we might have removed some sources of the variation (e.g. outliers) that often tends to hide phenomena of interest (see Pujol et al. 2005).

This is one of the few studies assessing HFCs within different types of populations and, to our knowledge, the first one assessing HFCs within contrasting plant populations in relation to anthropogenic habitat fragmentation (see Grueber et al. 2008). We acknowledge that it is clearly difficult to draw robust conclusions from only two large versus three small populations. However, and despite a lower statistical power, we found strong HFCs within large, as opposed to small myrtle populations. In addition, our data integrates the contemporary mating patterns, which provide us essential information to discuss the potential origin of differences in HFCs found between our study populations. We cannot completely rule out that a direct effect of polymorphic allozymes was behind the observed HFCs. Nevertheless, taken as a whole, our results suggest that a general effect (inbreeding) may better explain the observed HFCs and also that different genetic processes such as genetic drift and/or selection may have overridden this pattern within small populations (although these possibilities remain unexplored). Thus, our study points to a need for further attention to the genetic processes underlying among-population variation in HFCs and the complex relationships between heterozygosity in maternal plants and their progeny in relation to variation in the mating system. Our study is also a step towards understanding how genetic diversity of plant populations is maintained or lost within remnant habitat patches in a context where habitat loss and fragmentation is one of the critical drivers of global change.

Acknowledgments We are grateful to Sofia Vilaça Nora for her help and the 'Servicio de Invernadero de la Universidad de Sevilla' for its assistance in the greenhouse experiment. We are also very grateful to John R. Pannell and Patrice David whose helpful comments greatly improved earlier versions of this article. This study was funded by grants from the Spanish Ministerio de Educación y Ciencia (project CGL2008-000938/BOS) and the Andalusian Regional Government (Excellence Grants P06-RNM-01499, P07-RNM-02869 and P09-RNM-5280), and partially by a grant (HF2008-0040) for SpainFrance integrative actions from the MICINN programme.

\section{References}

Aguilar R, Quesada M, Ashworth L, Herrerías-Diego Y, Lobo J (2008) Genetic consequences of habitat fragmentation in plant populations: susceptible signals in plant traits and methodological approaches. Mol Ecol 17:5177-5188

Albaladejo RG, Fernández-Carrillo L, Aparicio A, Fernández-Manjarrés J, González-Varo JP (2009) Population genetic structure in Myrtus communis L. across a chronically fragmented landscape in the Mediterranean: can gene flow counteract habitat perturbation? Plant Biol 11:442-453

Amos W, Acevedo-Whitehouse K (2009) A new test for genotypefitness associations reveals a single microsatellite allele that strongly predicts the nature of tuberculosis infections in wild boar. Mol Ecol 9:1102-1111

Aparicio A (2008) Descriptive analysis of the 'relictual' Mediterranean landscape in the Guadalquivir River valley (southern Spain): a baseline for scientific research and the development of conservation action plans. Biodivers Conserv 17:2219-2232

Balloux F, Amos W, Coulson T (2004) Does heterozygosity estimate inbreeding in real populations? Mol Ecol 13:3021-3031

Bierne N, Tsitrone A, David P (2000) An inbreeding model of associative overdominance during a population bottleneck. Genetics 155:1981-1990

Britten HB (1996) Meta-analyses of the association between multilocus heterozygosity and fitness. Evolution 50:2158-2164

Byers DL, Waller DM (1999) Do plant populations purge their genetic load? Effects of population size and mating history on inbreeding depression. Ann Rev Ecol Syst 30:479-513

Chapman JR, Nakagawa S, Coltman DW, Slate J, Sheldon BC (2009) A quantitative review of heterozygosity-fitness correlations in animal populations. Mol Ecol 18:2746-2765 
Charlesworth B, Charlesworth D (1999) The genetic basis of inbreeding depression. Genet Res 74:329-340

Charlesworth D, Willis JH (2009) The genetics of inbreeding depression. Nature Rev Genetics 10:783-796

Coltman DW, Slate J (2003) Microsatellites measures of inbreeding: a meta-analyses. Evolution 57:971-983

David P (1998) Heterozygosity-fitness correlations: new perspective on old problems. Heredity 80:531-537

David P, Delay B, Jarne P (1997) Heterozygosity and growth in the marine bivalve Spisula ovalis: testing alternative hypothesis. Genet Res 70:215-223

Dudash MR, Carr DE, Fenster CB (1997) Five generations of enforced selfing and outcrossing in Mimulus guttatus: inbreeding depression variation at the population and family level. Evolution 51:54-65

Eckert CG, Kalisz S, Geber MA, Sargent R, Elle E, Cheptou PO et al (2010) Plant mating systems in a changing world. Trends Ecol Evol 25:35-43

Ellstrand NC, Elam DR (1993) Population genetic consequences of small population size: implications for plant conservation. Ann Rev Ecol Syst 24:217-243

González-Varo JP, Traveset A (2010) Among-individual variation in pollen limitation and inbreeding depression in a mixed-mating shrub. Ann Bot 106:999-1008

González-Varo JP, Arroyo J, Aparicio A (2009) Effects of fragmentation on pollinator assemblage, pollen limitation and seed production of Mediterranean myrtle (Myrtus communis). Biol Conserv 142:1058-1065

González-Varo JP, Albaladejo RG, Aparicio A, Arroyo J (2010) Linking genetic diversity, mating patterns and progeny performance in fragmented populations of a Mediterranean shrub. J Appl Ecol 47:1242-1252

González-Varo JP, Nora S, Aparicio A (2012) Bottlenecks for plant recruitment in woodland remnants: an ornithochorous shrub in a Mediterranean 'relictual' landscape. Persp Plant Ecol Evol Syst 14:111-122

Goodwillie C, Kalisz S, Eckert CG (2005) The evolutionary enigma of mixed mating systems in plants: occurrence, theoretical explanations, and empirical evidence. Ann Rev Ecol Syst 36:47-79

Grueber CE, Graham PW, Jamieson IG (2008) Heterozygosity-fitness correlations and their relevance to studies on inbreeding depression in threatened species. Mol Ecol 17:3978-3984
Hansson B, Westerberg L (2002) On the correlation between heterozygosity and fitness in natural populations. Mol Ecol 11: 2467-2474

Hansson B, Westerberg L (2008) Heterozygosity-fitness correlations within inbreeding classes: local or genome-wide effects? Conserv Genet 9:73-83

Karl SA, Avise JC (1992) Balancing selection at allozyme loci in oysters: implications from nuclear RFLPs. Science 256:100-102

Ledig FT, Guries RP, Bonefeld BA (1983) The relation of growth to heterozygosity in pitch pine. Evolution 37:1227-1238

Mitton JB (1997) Selection in natural populations. Oxford University Press, New York

Mitton JB, Grant MC (1984) Associations among protein heterozygosity, growth rate, and developmental homeostasis. Ann Rev Ecol Syst 15:479-499

Mitton JB, Schuster WSF, Cothran EG, De Fries J (1993) The correlation in heterozygosity between parents and their offspring. Heredity 71:59-63

Mueller JC, Hermisson J, Olano-Marin J, Hansson B, Kempenaers B (2010) Linking genetic mechanisms of heterozygosity-fitness correlations to footprints of selection at single loci. Evol Ecol 25:1-11

Pogson GH, Zouros E (1994) Allozyme and RFLP heterozygosities as correlates of growth in the scallop Placopecten magellanicus. Genetics 137:221-231

Pujol B, David P, McKey D (2005) Microevolution in agricultural environments: how a traditional Amerindian farming practice favours heterozygosity in cassava (Manihot esculenta Crantz, Euphorbiaceae). Ecol Letters 8:138-147

Ritland K (2002) Extensions of models for the estimation of mating systems using $\mathrm{n}$ independent loci. Heredity 88:221-228

Roach DA, Wulff RD (1987) Maternal effects in plants. Ann Rev Ecol Syst 18:209-235

Statsoft (2001) STATISTICA (data analysis software system), version 6. StatSoft, www.statsoft.com

Szulkin M, Bierne N, David P (2010) Heterozygosity-fitness correlations: a time for reappraisal. Evolution 64:1202-1217

Thelen GC, Allendorf FW (2001) Heterozygosity-fitness correlations in rainbow trout: effects of allozyme loci or associative overdominance? Evolution 55:1180-1187

Zouros E (1993) Associative overdominance: evaluating the effects of inbreeding and linkage disequilibrium. Genetica 89:35-46 